\title{
Efficacy and safety of nicorandil on perioperative myocardial injury in patients undergoing elective percutaneous coronary intervention: results of the PENMIPCI trial
}

This article was published in the following Dove Press journal:

Drug Design, Development and Therapy

\author{
Ziliang $\mathrm{Ye}^{1}$ \\ Haili Lu ${ }^{2}$ \\ Qiang Su' \\ Manyun Long' \\ Lang $\mathrm{Li}^{\prime}$ \\ On behalf of the PENMIPCI \\ investigators \\ 'Department of Cardiology, The First \\ Affiliated Hospital of Guangxi Medical \\ University, Nanning, Guangxi, People's \\ Republic of China; ${ }^{2}$ Department \\ of Orthodontics, The Affiliated \\ Dental Hospital of Guangxi Medical \\ University, Nanning, Guangxi, People's \\ Republic of China
}

Correspondence: Lang Li; Qiang Su Department of Cardiology, The First Affiliated Hospital of Guangxi Medical University, 6 Shuangyong Road, Qingxiu District, Nanning, Guangxi Zhuang Autonomous Region, 53002I, People's Republic of China

Tel +86 I 5277006843 Fax +86 I 370 77। 59I3

Email drrlilang@126.com; 3224014648@.com
Background: Previous studies have indicated that nicorandil can reduce perioperative myocardial injury (PMI) in patients undergoing elective percutaneous coronary intervention (ePCI), but this conclusion is still controversial. Additionally, studies reporting on the safety of nicorandil are lacking. Therefore, we performed this prospective study to evaluate the efficacy and safety of nicorandil on PMI in patients undergoing ePCI.

Methods: One hundred and forty-six patients with coronary heart disease (CHD) scheduled to undergo ePCI were randomly assigned to the nicorandil group $(n=74)$ or control group $(n=72)$. The primary outcomes were the change in cardiac troponin $\mathrm{T}(\mathrm{cTnT})$ and creatine kinase-MB (CK-MB) at 12 and 24 hours after surgery. The secondary outcome was the incidence of major adverse cardiac events (MACE), which was a composite of cardiac death, nonfatal myocardial infarction, new heart failure or coronary revascularization.

Results: There was no difference in age $(54.76 \pm 5.93$ vs $56.35 \pm 5.22)$ between the nicorandil group and the control group. In addition, no differences were observed in the cTnT and CK-MB levels between the two groups at admission (all $P>0.05$ ). Compared with those in the control group, the cTnT $(0.15 \pm 0.12$ vs $0.12 \pm 0.10$ at 12 hours and $0.17 \pm 0.12$ vs $0.13 \pm 0.10$ at 24 hours $)$ and CK-MB ( $15.35 \pm 8.23$ vs $12.31 \pm 7.93$ at 12 hours and $13.63 \pm 8.87$ vs $11.13 \pm 5.71$ at 24 hours) levels in the nicorandil group were significantly decreased after surgery (all $P<0.05$ ). Furthermore, nicorandil did not increase the incidence of MACE in the nicorandil group compared with the control group (12.16\% vs $12.50 \%)$.

Conclusions: Nicorandil can reduce PMI in patients undergoing ePCI and does not increase the incidence of MACE.

Clinical Trial Registration: URL: http://www.chictr.org.cn/. Unique Identifier: ChiCTRIOR-17012056.

Keywords: nicorandil, perioperative myocardial injury, elective percutaneous coronary intervention

\section{Introduction}

With the continuous development of the social economy, people's way of life has undergone profound changes. ${ }^{1}$ In addition, due to the aging of the population and the accelerated process of urbanization, the prevalence of cardiovascular disease risk factors is increasing year by year, which leads to an increasing number of cardiovascular diseases..$^{2-4}$ According to some research reports, cardiovascular disease is the leading cause of death among urban and rural residents in China..$^{5-7}$ Coronary heart 
disease (CHD) is one of the most common cardiovascular diseases, and its mortality has also been on the rise in recent years. ${ }^{8-10}$ In general, the increasing burden of cardiovascular diseases has become a major public health problem all over the world..$^{11-13}$

The results of a clinical study showed that percutaneous coronary intervention (PCI) is an effective way to treat CHD. ${ }^{14}$ However, periprocedural myocardial injury $(\mathrm{PMI})^{15,16}$ is a common complication of elective PCI. Previous studies have shown that PMI can directly reduce myocardial cell viability and cardiac function. ${ }^{17}$ Furthermore, PMI can lead to various serious events. ${ }^{18,19}$ Therefore, preventing PMI during elective PCI is a key concern of clinicians.

Nicorandil, an $N$-nicotinoyl nitrate drug, which can lead to diastolic coronary artery dilation, antagonizes platelet activation and eliminates inflammatory mediators, is used to treat various ischemic and cardiovascular diseases. ${ }^{16,20}$ Previous studies have shown that nicorandil used in PCI can improve myocardial perfusion and reduce the incidence of PMI. ${ }^{16,21,22}$ However, this conclusion is still controversial. Some scholars have noted that nicorandil can significantly prevent myocardial injury during PCI, ${ }^{21,23,24}$ while other scholars have indicated that nicorandil has no protective effect on myocardial injury. ${ }^{22}$ For example, Miyoshi et $\mathrm{al}^{25}$ found that intravenous use of nicorandil did not reduce the incidence of perioperative myocardial injury. In contrast, Isono et $\mathrm{al}^{26}$ noted that nicorandil significantly reduced the incidence of perioperative myocardial injury and improved left ventricular wall motion. Interestingly, our previous meta-analysis indicated that nicorandil reduced PMI and reduced the incidence of adverse reactions in Chinese patients following elective PCI, but this phenomenon was not obvious for nonChinese patients. ${ }^{16}$ Therefore, we performed the PENMIPCI (Protective Effects of Nicorandil on Myocardial Injury after Percutaneous Coronary Intervention) trial to evaluate the efficacy and safety of nicorandil on PMI in patients undergoing elective PCI.

\section{Methods}

The data, analytic methods, and study materials will not be made immediately available to other researchers for the purposes of reproducing the results or replicating the procedure. Requests for these materials can be sent to the corresponding author (LL), and if the applicant is approved as qualified for access to the data, we will provide the data as well as information regarding the analytic methods and study materials.

\section{Study design}

The PENMIPCI trial is a single-center, randomized, open-label trial conducted at the First Affiliated Hospital of Guangxi Medical University, Nanning, Guangxi, China. The detailed design of the study has been described at http://www.chictr. org.cn/. The flow chart for this study is shown in Figure 1. In summary, a total of 164 patients who underwent elective PCI were included in this study. Ultimately, 146 patients who underwent elective PCI were randomly assigned to two groups after providing informed consent: the control group (who received aspirin $100 \mathrm{mg}$ daily + clopidogrel $75 \mathrm{mg}$ daily) and the nicorandil group (who received aspirin $100 \mathrm{mg}$ daily + clopidogrel $75 \mathrm{mg}$ daily + nicorandil $5 \mathrm{mg}$,

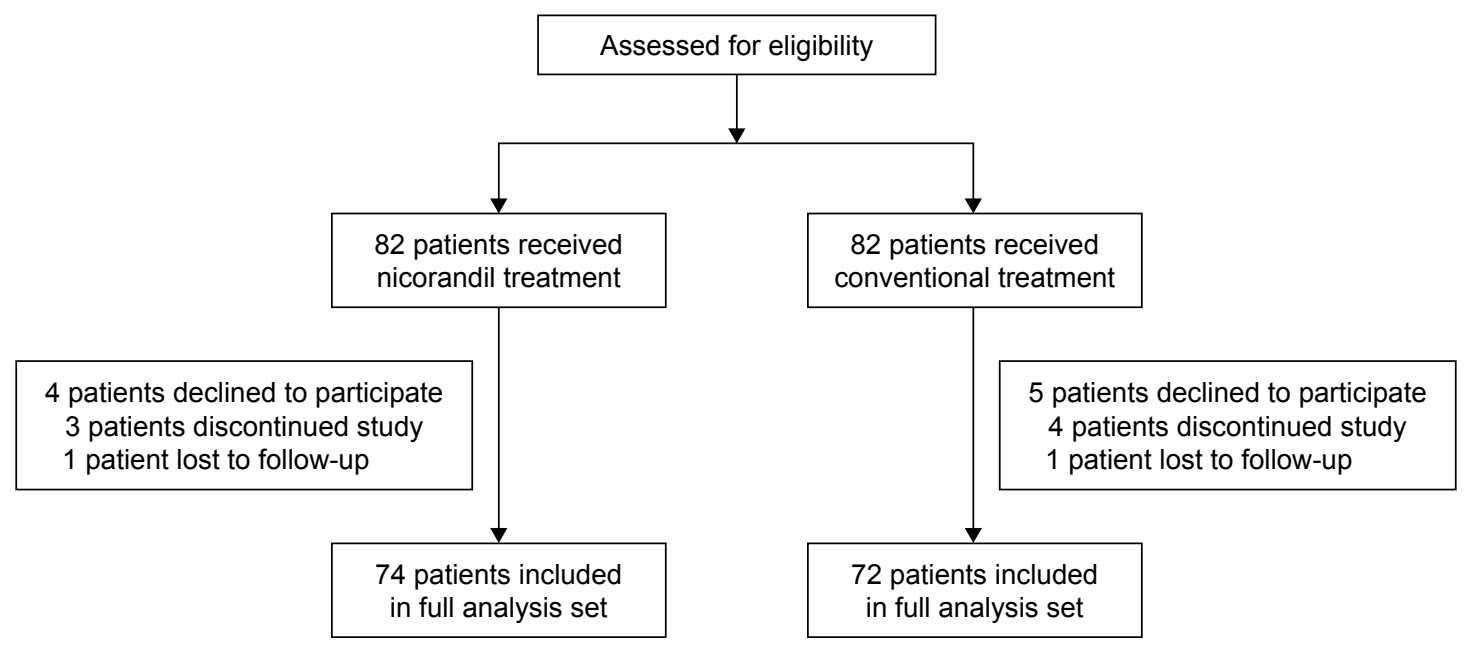

Figure I Study flow chart. 
three times a day). The use of nicorandil was as follows: $5 \mathrm{mg}$ of nicorandil was taken orally for 3 days before PCI and 7 days after PCI. All patients were given beta-blockers, angiotensin-converting enzyme inhibitors, or angiotensin II receptor blockers and statins according to the current guidelines. Data analysis was carried out independently by the researchers. The corresponding authors (Lang Li and Qiang $\mathrm{Su}$ ) had the right to access all study data and bore the ultimate responsibility for the decision to submit the data for publication. This study was approved by the ethical committee of the First Affiliated Hospital of Guangxi Medical University (approval number: 2017 (KY-E-026; http://www. chictr.org.cn/). All participants were informed of the study and provided written informed consent. In addition, this study was conducted in accordance with the tenets of the Declaration of Helsinki.

\section{Study patients}

The inclusion criteria were as follows: patients with CHD scheduled for elective PCI at the First Affiliated Hospital of Guangxi Medical University; aged 18 to 75 years old; male or non-pregnant females; coronary blood flow of more than TIMI2 (thrombolysis in myocardial infarction, TIMI) after PCI; and no potassium channel openers within 1 month before enrollment. The exclusion criteria were as follows: acute coronary syndrome (including unstable angina, ST-segment elevation myocardial infarction and non-ST-segment elevation myocardial infarction); severe cardiac insufficiency (New York Heart Association $[\mathrm{NYHA}] \geq$ III class or left ventricular ejection fraction [LVEF] less than $40 \%$ as measured by echocardiography); severe liver and renal insufficiency (estimated glomerular filtration rate $\leq 30 \%$ ); bleeding tendency; gastrointestinal bleeding; history of a malignant tumor, such as lung cancer, primary liver cancer and digestive tract malignant tumors; allergy to antiplatelet drugs or anticoagulant drugs; scheduled coronary artery bypass grafting treatment; severe valvular heart diseases; autoimmune diseases; and potassium channel medication use within 1 month of enrollment. Other detailed descriptions can be found at http://www. chictr.org.cn/.

\section{Randomization}

Patients who met the inclusion criteria and exclusion criteria mentioned above were randomly assigned in a 1:1 fashion to the control group and nicorandil group using a random number table. This process was entirely conducted by the researchers, and no pharmacist or pharmaceutical company were involved in this process.

\section{Study endpoints and follow-up}

The primary outcomes were the change in cardiac troponin $\mathrm{T}$ (cTnT) and creatine kinase-MB (CK-MB) at 12 and 24 hours after surgery. The secondary outcome was the incident of major adverse cardiac events (MACE) 30 days after the operation, which was a composite of cardiac death, nonfatal myocardial infarction, new heart failure or coronary revascularization. The serious adverse events mentioned above were checked and confirmed by two cardiovascular specialists according to the guidelines that were formulated. All patients were followed-up by telephone at 30 days after the operation.

\section{Covariant variables}

We used the electronic medical records of the First Affiliated Hospital of Guangxi Medical University to collect the demographic characteristics, comorbidities and cardiac medications of all patients. The patient information included age, sex, diabetes, hypertension, dyslipidemia, smoking status, weight, height, body mass index (BMI), estimated glomerular filtration rate (eGFR), glutamic-oxaloacetic transaminase, alanine aminotransferase, low-density lipoprotein, highdensity lipoprotein, total cholesterol, triglycerides, uric acid, fasting blood glucose, hemoglobin A1C, brain natriuretic peptide, left ventricular end diastolic diameter (LVDD) and LVEF. The variables mentioned above were measured within 24 hours after admission. Echocardiography was usually performed within 24 hours after admission by two echocardiography experts.

\section{Treatment and procedure}

The drugs used before and after PCI were given in accordance with the accepted guidelines and practice standards. The PCI procedure and perioperative anticoagulant therapy were carried out in accordance with the accepted guidelines.

\section{Statistical analysis}

The hypothesis of this study is that nicorandil can reduce the incidence of PMI in patients undergoing ePCI compared with the control group. In our preliminary experiment, the incidence of PMI in the control group was $43.6 \%$, while the incidence of PMI in the nicorandil group was $27.4 \%$. With a two-sided alpha level of $5 \%$ and beta error of $20 \%$, each group needed to recruit 66 participants. In addition, supposing that $10 \%$ of the participants in each group would be lost 
or withdraw from the study, each group ultimately needed to include 73 participants.

In this study, the analysis was based on intention-totreat principles, and all patients involved in the study were included in the final analysis. The results are presented as the mean \pm standard deviation or number + percent. Categorical variables were compared using the chi-squared test. We compared the mean values of continuous variables between the two groups by unpaired Student's $t$-test. Differences in the mean values of cTnT and CK-MB between baseline and 12 and 24 hours after operation were compared using repeated-measures analysis of variance.

The incidence of MACE events between the two groups was compared by multivariate logistic regression analyses with hazard ratios (HRs) and 95\% confidence intervals (CIs). In addition, the Kaplan-Meier curve method and log-rank test were used in this study to compare the survival time of the two groups. We used the Cox proportional hazards model to estimate HRs between the two groups while adjusting for potential confounding factors, including age, sex, diabetes, hypertension, dyslipidemia, smoking status, weight, height, BMI, eGFR, glutamic-oxaloacetic transaminase, alanine aminotransferase, low-density lipoprotein, high-density lipoprotein, total cholesterol, triglycerides, uric acid, fasting blood glucose, hemoglobin A1C, brain natriuretic peptide, LVDD and LVEF.

Furthermore, we also carried out a subgroup analysis. All patients were stratified into six groups according to age (age $>65$ years or age $<65$ years), sex (female or male), diabetes (yes or no), hypertension (yes or no), dyslipidemia (yes or no) and smoking status (yes or no). All reported probability values were two-sided with a $P$-value $<0.05$ considered to be statistically significant. Data analysis was performed using IBM SPSS Statistics for Windows, version 22.0 (IBM Corporation, Armonk, NY, USA), R statistical software

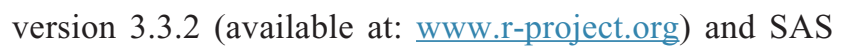
software (SAS Institute Inc., Cary, NC, USA), version 9.2.

\section{Results}

\section{Study flow chart}

From October 2017 through April 2018, 164 patients were recruited in the present study; 82 patients were assigned to the nicorandil group and 82 patients were assigned to the control group. During this study, four patients declined to participate, three patients discontinued the study, and one patient was lost to follow-up in the nicorandil group; five patients declined to participate, four patients discontinued the study, and one patient was lost to follow-up in the control group.
Ultimately, 74 patients were included in the full analysis set in the nicorandil group and 72 patients were included in the full analysis set in the control group. The flowchart is shown in Figure 1.

\section{Baseline characteristics of study participants}

There was no significant difference in the baseline characteristics between the two groups (Table 1). The average age of the control group was $56.35 \pm 5.22$ years, while the average age of the nicorandil group was $54.76 \pm 5.93$ years $(P=0.088)$. Females accounted for $25 \%$ of the control group and $24.32 \%$ of the nicorandil group $(P=0.925)$. Additionally, no significant differences were observed between the two groups in terms of drug application and criminal vessel (Table 1).

\section{Primary outcome}

The primary outcomes of this study were the changes in cTnT and CK-MB at 12 and 24 hours after surgery. There was no significant difference in cTnT $(0.032 \pm 0.018$ for the control group and $0.030 \pm 0.022$ for the nicorandil group, $P=0.549)$ or $\mathrm{CK}-\mathrm{MB}(4.64 \pm 2.78$ for the control group and $4.35 \pm 2.48$ for the nicorandil group, $P=0.501$ ) between the two groups at admission (Table 2). The levels of cTnT in the nicorandil group decreased significantly compared with those of the control group at both 12 hours $(0.12 \pm 0.10$ vs $0.15 \pm 0.12, P=0.012)$ and 24 hours $(0.13 \pm 0.10$ vs $0.17 \pm 0.12$, $P=0.030$ ) after surgery (Table 2 and Figure 2). Similarly, the levels of CK-MB in the nicorandil group also decreased significantly compared with those of the control group at both 12 hours $(12.31 \pm 7.93$ vs $15.35 \pm 8.23, P=0.024)$ and 24 hours ( $11.13 \pm 5.71$ vs $13.63 \pm 8.87, P=0.022$ ) after surgery (Table 2 and Figure 3 ).

\section{Secondary outcome}

During a mean follow-up of $24.12 \pm 3.00$ days, nine patients $(9 / 72,12.50 \%)$ had MACE events in the control group and nine patients $(9 / 74,12.16 \%)$ had MACE events in the nicorandil group. A Cox proportional hazards model showed that no significant difference was observed in MACE events between the two groups $(\mathrm{HR}=0.99,95 \% \mathrm{CI}$ : 0.39-2.55, $P=0.98$ ) (Figure 4).

\section{Subgroup analyses of the secondary outcome among the two groups}

In subgroup analyses, we found that nicorandil did not increase the incidence of MACE events, regardless of 
Table I Baseline characteristics of two groups

\begin{tabular}{|c|c|c|c|}
\hline Characteristic & $\begin{array}{l}\text { The control } \\
\text { group }\end{array}$ & $\begin{array}{l}\text { The nicorandil } \\
\text { group }\end{array}$ & $P$-value \\
\hline Total participants & 72 & 74 & \\
\hline Age (years) & $56.35 \pm 5.22$ & $54.76 \pm 5.93$ & 0.088 \\
\hline Sex, female (\%) & $18(25.00)$ & $18(24.32)$ & 0.925 \\
\hline Diabetic, yes (\%) & $33(45.83)$ & $38(5 \mathrm{I} .35)$ & 0.505 \\
\hline Hypertension, yes (\%) & $66(91.67)$ & $62(83.78)$ & 0.147 \\
\hline Dyslipidemia, yes (\%) & 57 (79.17) & $60(81.08)$ & 0.772 \\
\hline Smoking, yes (\%) & $4 \mathrm{I}(56.94)$ & $46(62.16)$ & 0.521 \\
\hline Weight (kg) & $60.13 \pm 11.11$ & $63.84 \pm 10.57$ & $0.04 I$ \\
\hline Height $(\mathrm{cm})$ & $158.38 \pm 8.72$ & $160.21 \pm 8.00$ & 0.187 \\
\hline BMI $\left(\mathrm{kg} / \mathrm{m}^{2}\right)$ & $23.86 \pm 3.26$ & $24.82 \pm 3.35$ & 0.083 \\
\hline Heart rate (bpm) & $78 \pm 12$ & $80 \pm 13$ & 0.336 \\
\hline Systolic pressure $(\mathrm{mmHg})$ & $132 \pm 24$ & $130 \pm 22$ & 0.600 \\
\hline Diastolic pressure $(\mathrm{mmHg})$ & $72 \pm 12$ & $74 \pm 11$ & 0.295 \\
\hline White blood cell (109/L) & $6.12 \pm 0.67$ & $6.24 \pm 0.74$ & 0.306 \\
\hline High sensitive-C-reactive protein $(\mathrm{mg} / \mathrm{dL})$ & $0.43 \pm 0.04$ & $0.44 \pm 0.04$ & 0.133 \\
\hline eGFR (mL/min) & $62.23 \pm 14.10$ & $60.36 \pm 19.60$ & 0.510 \\
\hline Glutamic-oxalacetic transaminase (U/L) & $24.19 \pm 8.54$ & $24.72 \pm 11.29$ & 0.754 \\
\hline Alanine aminotransferase (U/L) & $20.19 \pm 10.31$ & $22.11 \pm 12.20$ & 0.308 \\
\hline Low-density lipoprotein (mg/dL) & $87.75 \pm 31.69$ & $87.06 \pm 30.69$ & 0.894 \\
\hline High-density lipoprotein (mg/dL) & $42.74 \pm 15.83$ & $44.30 \pm 17.00$ & 0.568 \\
\hline Total cholesterol (mg/dL) & I $55.24 \pm 47.04$ & $|54.3| \pm 5 \mid .85$ & 0.910 \\
\hline Triglyceride (mg/dL) & $123.24 \pm 78.54$ & $134.62 \pm 82.07$ & 0.393 \\
\hline Uric acid (mg/dL) & $5.33 \pm 1.90$ & $5.43 \pm 2.00$ & 0.769 \\
\hline Fasting blood glucose $(\mathrm{mg} / \mathrm{dL})$ & $125.06 \pm 7 \mid .53$ & $117.23 \pm 67.09$ & 0.496 \\
\hline Hemoglobin AIC (\%) & $5.6 I \pm 2.0 I$ & $5.53 \pm 2.01$ & 0.814 \\
\hline Brain natriuretic peptide $(\mathrm{pg} / \mathrm{mL})$ & $85.64 \pm 197.99$ & $95.17 \pm 168.27$ & 0.754 \\
\hline LVDD (mm) & $45.92 \pm 11.05$ & $45.60 \pm 12.63$ & 0.874 \\
\hline LVEF (\%) & $59.56 \pm 15.99$ & $60.00 \pm 17.23$ & 0.876 \\
\hline Aspirin, yes (\%) & $72(100.00)$ & $74(100.00)$ & 0.869 \\
\hline Clopidogrel, yes (\%) & $71(98.61)$ & $70(94.59)$ & 0.182 \\
\hline Beta-blockers, yes (\%) & $50(69.44)$ & $42(56.76)$ & 0.112 \\
\hline ACEI, yes $(\%)$ & $64(88.89)$ & $68(91.89)$ & 0.266 \\
\hline ARB, yes (\%) & $31(43.06)$ & $39(52.70)$ & 0.243 \\
\hline Statin, yes (\%) & $54(75.00)$ & $58(78.38)$ & 0.629 \\
\hline RCA, yes (\%) & $47(65.28)$ & $46(62.16)$ & 0.696 \\
\hline LAD, yes (\%) & $37(51.39)$ & $42(56.76)$ & 0.515 \\
\hline LCX, yes (\%) & $56(77.78)$ & $53(71.62)$ & 0.393 \\
\hline AHA-ACC classification, yes (\%) & & & 0.928 \\
\hline Type A & $17(23.6)$ & $19(25.7)$ & \\
\hline Type BI & $24(33.3)$ & $23(31.1)$ & \\
\hline Type B2 & $26(36.1)$ & $25(33.8)$ & \\
\hline Type C & $5(7.0)$ & $7(9.4)$ & \\
\hline Number of stents, yes (\%) & $2.1 \pm 0.10$ & $2.1 \pm 0.11$ & 1.0 \\
\hline Contrast medium (mL) & $102 \pm 13$ & $104 \pm 12$ & 0.335 \\
\hline Stent length (mm) & $18 \pm 1.3$ & $|8 \pm 1|$. & 1.0 \\
\hline Coronary heart disease & & & 0.729 \\
\hline Stable angina & $32(44.4)$ & $35(47.3)$ & \\
\hline Unstable angina & $40(55.6)$ & $39(52.7)$ & \\
\hline
\end{tabular}

Note: Data shown as mean \pm standard deviation or $\mathrm{n}(\%)$.

Abbreviations: ACEI, angiotensin-converting enzyme inhibitor; AHA-ACC, American College of Cardiology-American Heart Association; ARB, angiotensin II receptor antagonist; BMI, body mass index; eGFR, estimated glomerular filtration rate; LAD, left anterior descending branch; LCX, left circumflex branch; LVDD, left ventricular internal dimension diastole; LVEF, left ventricular ejection fraction; RCA, right coronary artery.

age (age $>65$ years: HR: $-0.49,95 \%$ CI: -2.21 to 1.17 ; age $<65$ years; HR: $0.03,95 \%$ CI: -1.12 to 1.28 ), sex (female: HR: $0.11,95 \% \mathrm{CI}:-2.04$ to 1.03 ; male: HR: -0.04 , 95\% CI: -1.17 to 1.08 ), diabetes (with diabetes; HR: -0.17 ,
95\% CI: -1.43 to 1.07 ; without diabetes; HR: $0.09,95 \%$ CI: -1.56 to 1.76 ), hypertension (with hypertension: HR: $-0.58,95 \% \mathrm{CI}:-1.71$ to 0.57 ; without hypertension: HR: $-0.14,95 \%$ CI: -0.62 to 0.49 ), dyslipidemia (with 
Table 2 The levels of cardiac troponin T and creatine kinase-MB (CK-MB) at admission, 12 hours and 24 hours after operation

\begin{tabular}{|c|c|c|c|}
\hline Characteristic & $\begin{array}{l}\text { The control } \\
\text { group }\end{array}$ & $\begin{array}{l}\text { The nicorandil } \\
\text { group }\end{array}$ & $P$-value \\
\hline Total participants & 72 & 74 & \\
\hline Cardiac troponin $\mathrm{T}$ at admission $(\mu \mathrm{g} / \mathrm{L})$ & $0.032 \pm 0.018$ & $0.030 \pm 0.022$ & 0.549 \\
\hline CK-MB at admission (U/L) & $4.64 \pm 2.78$ & $4.35 \pm 2.48$ & $0.50 \mathrm{I}$ \\
\hline Cardiac troponin $\mathrm{T}$ after 12 hours $(\mu \mathrm{g} / \mathrm{L})$ & $0.15 \pm 0.12$ & $0.12 \pm 0.10$ & 0.012 \\
\hline CK-MB after 12 hours (U/L) & $15.35 \pm 8.23$ & $|2.3| \pm 7.93$ & 0.024 \\
\hline Cardiac troponin T after 24 hours $(\mu g / L)$ & $0.17 \pm 0.12$ & $0.13 \pm 0.10$ & 0.030 \\
\hline CK-MB after 24 hours (U/L) & $13.63 \pm 8.87$ & $|1.13 \pm 5.7|$ & 0.022 \\
\hline
\end{tabular}

Note: Data shown as number or mean \pm standard deviation.

dyslipidemia: HR: $0.15,95 \% \mathrm{CI}:-1.11$ to 1.39 ; without dyslipidemia: HR: $-0.29,95 \% \mathrm{CI}:-1.97$ to 1.43 ) and smoking status (current smoker: HR: $-0.14,95 \% \mathrm{CI}:-1.27$ to 1.03 ; non-smoker: HR: 0.11, 95\% CI: -1.90 to 2.14) (Figure 5).

\section{Discussion}

\section{Main findings}

In this single-center, randomized, open-label trial, we found that nicorandil significantly reduced the incidence of PMI, mainly the levels of cTnT and CK-MB at 12 and 24 hours after ePCI surgery. In addition, our results also indicated that nicorandil did not increase the incidence of MACE (a composite of cardiac death, nonfatal myocardial infarction, new heart failure or coronary revascularization) within 30 days $(12.16 \%$ in the nicorandil group and $12.50 \%$ in the control group). Furthermore, this result remained consistent in subgroup analysis. Our findings support the hypothesis that nicorandil can reduce PMI in patients undergoing ePCI and does not increase the incidence of MACE.

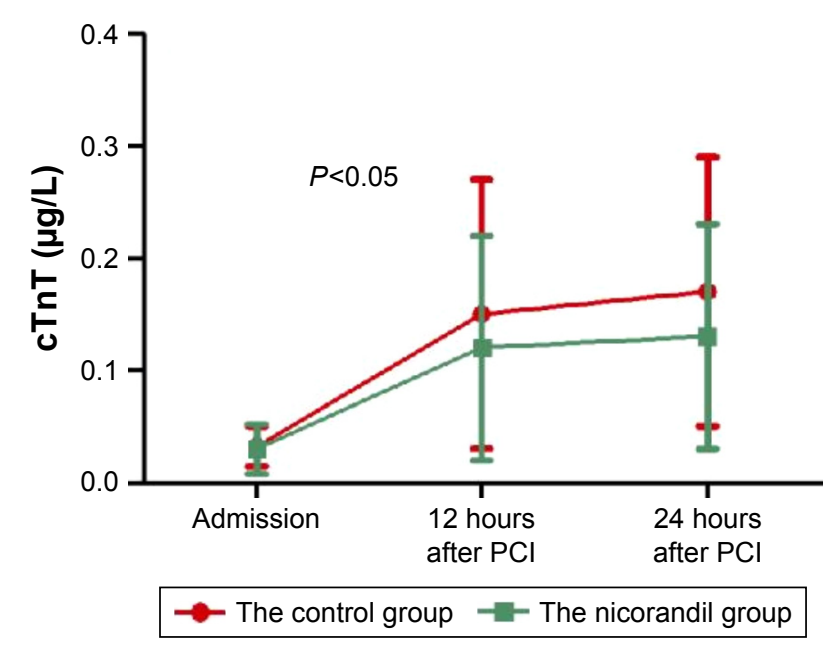

Figure 2 Levels of cardiac troponin $\mathrm{T}(\mathrm{cTnT})$ at admission and 12 hours and 24 hours after the operation.

Abbreviation: $\mathrm{PCl}$, percutaneous coronary intervention.

\section{Comparison with previous studies}

Previously, a large number of studies have reported the relationship between nicorandil and PMI in patients undergoing ePCI. However, there is controversy regarding this conclusion. Some scholars support the hypothesis that nicorandil can reduce the incidence of myocardial injury, while the results of other scholars do not support this conclusion. For instance, Hwang et $\mathrm{al}^{23}$ carried out a clinical study including 41 patients in the nicorandil group and 40 patients in the control group and found that nicorandil did not reduce the incidence of PMI in patients with stable or unstable angina. In addition, another study observed the clinical effect of intravenous nicorandil in preventing perioperative myocardial injury in the Japanese population. The results also support the hypothesis that nicorandil can prevent perioperative myocardial injury. ${ }^{27}$ Similarly, another subgroup analysis conducted in diabetic patients demonstrated that nicorandil reduced perioperative myocardial injury in patients undergoing ePCI. ${ }^{24}$ Interestingly, the findings also suggested that nicorandil improved left ventricular ejection

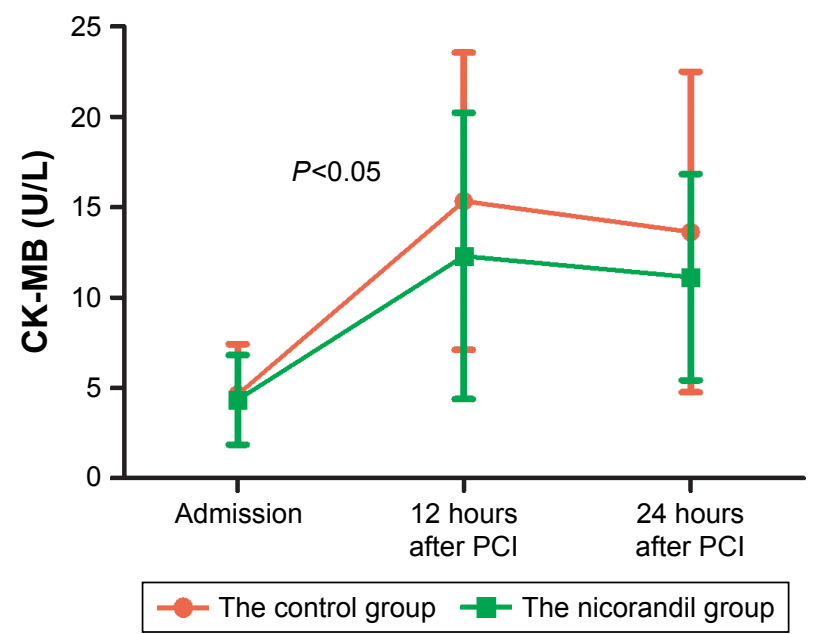

Figure 3 Levels of creatine kinase-MB (CK-MB) at admission and 12 and 24 hours after the operation.

Abbreviation: $\mathrm{PCl}$, percutaneous coronary intervention. 


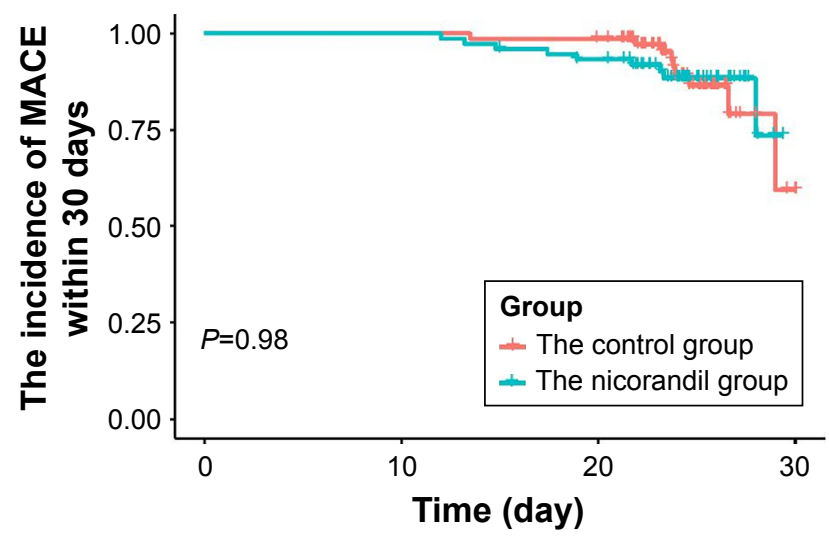

Figure 4 Incidence of MACE events within 30 days after ePCl surgery between the control group and nicorandil group.

Abbreviations: MACE, major adverse cardiac events; ePCl, elective percutaneous coronary intervention.

fraction after 6 months $(63.5 \% \pm 7.7 \%$ vs $56.5 \% \pm 8.3 \%)$. By contrast, some scholars hold the opposite opinion. A study conducted in South Korea found that an intracoronary injection of nicorandil did not reduce the levels of CK-MB and troponin I, regardless of whether patients had stable or unstable angina. Interestingly, another study conducted in the same country (Republic of Korea) found the opposite result. ${ }^{22}$ Since the issue of whether nicorandil can prevent perioperative myocardial injury is controversial, we previously conducted a systematic review and meta-analysis; ${ }^{16} 16$ studies were included in our meta-analysis. Surprisingly, our findings showed that nicorandil was effective for the Chinese population, but not for the non-Chinese population.

In general, it remains controversial as to whether nicorandil can prevent perioperative myocardial injury in patients undergoing ePCI. Therefore, we performed this randomized controlled trial to explore the preventive effect of nicorandil on myocardial injury. The PENMIPCI trial is a single-center, randomized, open-label trial conducted in Guangxi, China. The result of the PENMIPCI trial found that the $\mathrm{cTnT}$ and CK-MB levels at 12 and 24 hours were significantly decreased in the nicorandil group compared with those of the control group (Table 2) following ePCI surgery. In addition, our results indicated that nicorandil did not increase the incidence of adverse cardiovascular events within 30 days (Figure 3). Similarly, no significant change was found in subgroup analyses (Figure 4).

The results of the PENMIPCI trial are partly contradictory to previous studies, and the possible explanations are

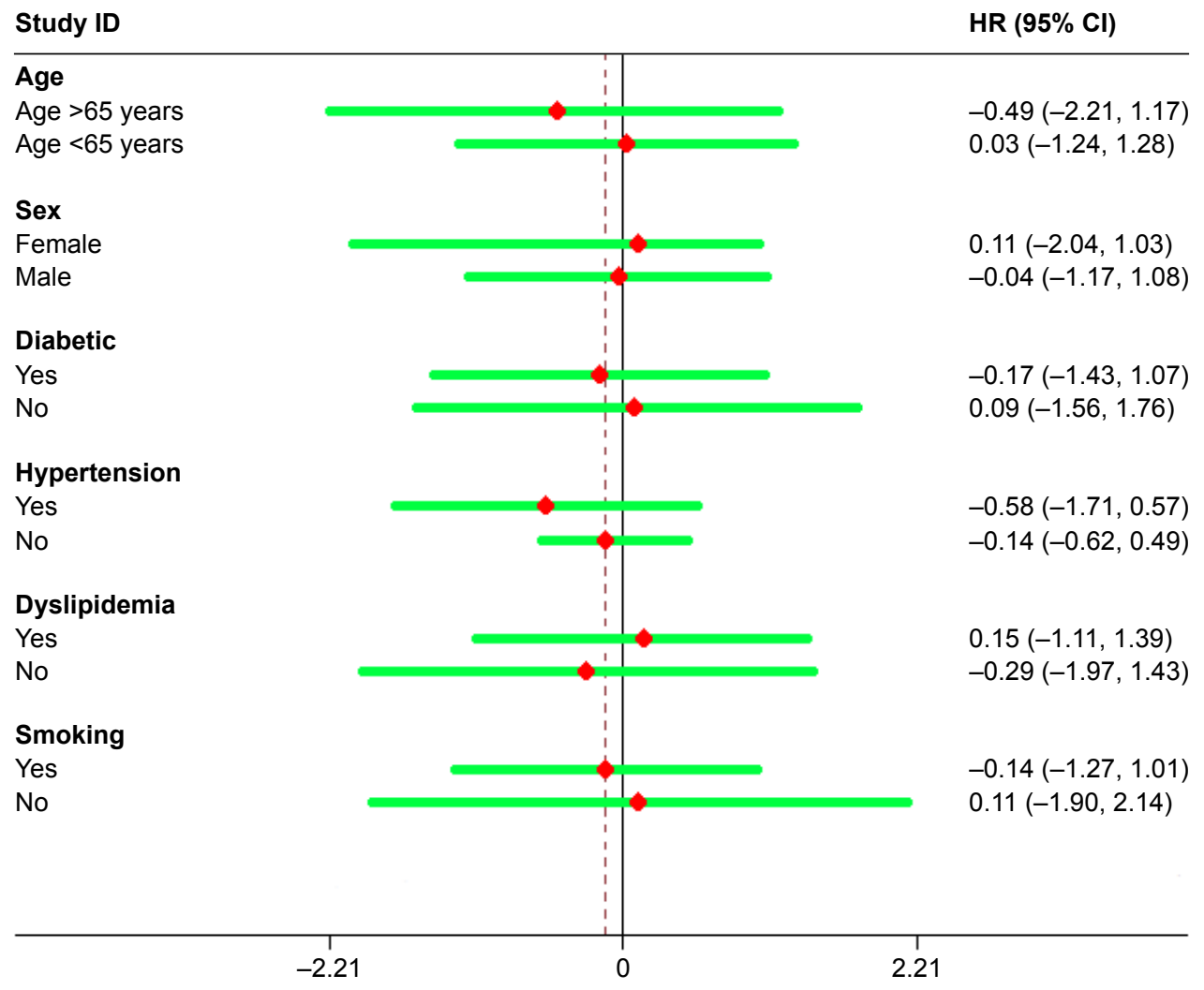

Figure $\mathbf{5}$ Incidence of MACE events between the control group and nicorandil group in subgroup analyses. Abbreviations: MACE, major adverse cardiac events; HR, hazard ratio. 
as follows. First, there is a great variety in the drug delivery methods used for nicorandil. Some studies have used intracoronary injections of nicorandil, ${ }^{22,25}$ while others have been carried out using oral administration. ${ }^{28}$ The different administration methods of nicorandil may lead to different results. Second, the difference in dosage is a possible reason leading to the different results. In some studies, the dosage of nicorandil was $4 \mathrm{mg}$ once daily, ${ }^{21,23}$ while in other studies, the dosage of nicorandil was $20 \mathrm{mg}$ once daily. ${ }^{24}$ Third, there are also significant differences in the follow-up period over which major adverse cardiovascular events were observed. The observation time for some studies was 1 month, 6 months, or even 1 year. Fourth, some previous studies did not describe the grouping method in detail, which is one of the reasons leading to a mixed bias. In the PENMIPCI trial, all patients were randomly assigned to the control group and nicorandil group according to the random number table method. Fifth, differences in the research populations may be an important reason leading to inconsistent results, and this hypothesis can be confirmed by our previous meta-analysis. As is known, the adaptability and metabolic rate of drugs vary among different populations.

\section{Strength and weakness}

Our study has several strengths. First, the analysis of the PENMIPCI trial was based on intention-to-treat principles, which is notably different from previous studies. In clinical practice, patients have the right to choose suitable treatment methods, and intention-to-treat analysis is more in line with the real world. In this case, we can minimize the confounding bias and reach a conclusion that is more consistent with the real world. Second, as a randomized controlled trial, the baseline information of the PENMIPCI trial was well balanced, with no significant differences between the groups. Under these circumstances, we were able to control the confounding factors to the minimum, thus increasing the reliability of the results. Third, subgroup analyses were also performed in the PENMIPCI trial according to age (age $>65$ years or age $<65$ years), sex, diabetes, hypertension, dyslipidemia and smoking status. The results of the subgroup analyses did not change our conclusions, thus indicating the robustness of the PENMIPCI trial.

Our study also has several limitations. First, as a singlecenter study, whether the results of the PENMIPCI trial can be applied to other regions or countries still needs to be confirmed in further research. Second, nicorandil was administered orally in the PENMIPCI trial. Thus, it should be noted that the results of this study only indicate that oral nicorandil can significantly reduce the incidence of myocardial injury during the perioperative period and cannot represent the effect of intracoronary nicorandil. It is possible that the route of administration of nicorandil might affect the research results.

\section{Conclusion}

Our results suggest that nicorandil can reduce $\mathrm{PMI}$ in patients undergoing ePCI without increasing the incidence of MACE. The results are consistent in the subgroup analyses. Considering the difference in dosage and route of administration, this result still requires further confirmation.

\section{Acknowledgments}

This study was supported by grants from the National Natural Science Foundation of China (grant number 81600283) and the Guangxi Natural Science Foundation (grant number 2016GXNSFBA380022).

\section{Disclosure}

The authors report no conflicts of interest in this work.

\section{References}

1. Fuenmayor A, Granell R, Tortosa MA Ángeles. Caring for older people: an analysis of the small business sector. The Service Industries Journal. 2012;32(15):2347-2363.

2. Lam NN, Kim SJ, Knoll GA, et al. The risk of cardiovascular disease is not increasing over time despite aging and higher comorbidity burden of kidney transplant recipients. Transplantation. 2016;101(3):588-596.

3. Gada H, Kirtane AJ, Newman W, et al. 5-year results of a randomized comparison of XIENCE V everolimus-eluting and TAXUS paclitaxeleluting stents: final results from the SPIRIT III trial (clinical evaluation of the XIENCE V everolimus eluting coronary stent system in the treatment of patients with de novo native coronary artery lesions). JACC Cardiovasc Interv. 2013;6(12):1263-1266.

4. Meng M, Gao B, Wang X, Bai ZG, Sa RN, Ge B. Long-term clinical outcomes of everolimus-eluting stent versus paclitaxel-eluting stent in patients undergoing percutaneous coronary interventions: a metaanalysis. BMC Cardiovasc Disord. 2016;16:34.

5. Zhou B, Wu Y, Yang J, Li Y, Zhang H, Zhao L. Overweight is an independent risk factor for cardiovascular disease in Chinese populations. Obes Rev. 2002;3(3):147-156.

6. He Y, Jiang B, Wang J, et al. BMI versus the metabolic syndrome in relation to cardiovascular risk in elderly Chinese individuals. Diabetes Care. 2007;30(8):2128-2134.

7. Hou F, Jiang J, Chen J, et al. China collaborative study on dialysis: a multi-centers cohort study on cardiovascular diseases in patients on maintenance dialysis. BMC Nephrol. 2012;13:94.

8. Marenberg ME, Risch N, Berkman LF, Floderus B, de Faire U. Genetic susceptibility to death from coronary heart disease in a study of twins. N Engl J Med Overseas Ed. 1994;330(15):1041-1046.

9. Bendinelli B, Masala G, Saieva C, et al. Fruit, vegetables, and olive oil and risk of coronary heart disease in Italian women: the EPICOR Study. Am J Clin Nutr. 2011;93(2):275-283.

10. Rachas A, Raffaitin C, Barberger-Gateau P, et al. Clinical usefulness of the metabolic syndrome for the risk of coronary heart disease does not exceed the sum of its individual components in older men and women. The Three-City (3C) Study. Heart. 2012;98(8):650-655. 
11. Mensah GA, Moran AE, Roth GA, Narula J. The global burden of cardiovascular diseases, 1990-2010. Glob Heart. 2014;9(1):183-184.

12. Roth GA, Johnson C, Abajobir A, et al. Global, regional, and national burden of cardiovascular diseases for 10 causes, 1990 to 2015. $\mathrm{J} \mathrm{Am}$ Coll Cardiol. 2017;70(1):1-25.

13. Smith SC Jr, Chen D, Collins A, et al. Moving from political declaration to action on reducing the global burden of cardiovascular diseases: a statement from the global cardiovascular disease taskforce. Circulation. 2013;128:2546-2548. Erratum: e450.

14. Fortuna D, Nicolini F, Guastaroba P, et al. Coronary artery bypass grafting vs percutaneous coronary intervention in a 'real-world' setting: a comparative effectiveness study based on propensity score-matched cohorts. Eur J Cardio-Thoracic Surg. 2013;44(1):e16-e24.

15. Cuculi F, Lim CC, Banning AP. Periprocedural myocardial injury during elective percutaneous coronary intervention: is it important and how can it be prevented? Heart. 2010;96(10):736-740.

16. Ye Z, Su Q, Li L. The clinical effect of nicorandil on perioperative myocardial protection in patients undergoing elective PCI: A systematic review and meta-analysis. Sci Rep. 2017;7:45117.

17. Nagele $P$, Liggett SB. Genetic variation, $\beta$-blockers, and perioperative myocardial infarction. Anesthesiology. 2011;115(6):1316-1327.

18. Kim SE, Park DG, Lee JH, Han KR, Oh DJ. Utility of B-type natriuretic peptide for predicting perioperative cardiovascular events in patients without history of cardiovascular disease undergoing major non-cardiac surgery. Korean Circ J. 2011;41(1):11-15.

19. Cuthbertson BH, McKeown A, Croal BL, Mutch WJ, Hillis GS. Utility of B-type natriuretic peptide in predicting the level of peri- and postoperative cardiovascular support required after coronary artery bypass grafting. Crit Care Med. 2005;33(2):437-442.
20. Horinaka S. Use of nicorandil in cardiovascular disease and its optimization. Drugs. 2011;71(9):1105-1119.

21. Kim JH, Jeong MH, Yun KH, et al. Myocardial protective effects of nicorandil during percutaneous coronary intervention in patients with unstable angina. Circ J. 2005;69(3):306-310.

22. Kim SJ, Kim W, Woo JS, et al. Effect of myocardial protection of intracoronary adenosine and nicorandil injection in patients undergoing non-urgent percutaneous coronary intervention: a randomized controlled trial. Int J Cardiol. 2012;158(1):88-92.

23. Hwang J, Lee HC, Kim BW, et al. Effect on periprocedural myocardial infarction of intra-coronary nicorandil prior to percutaneous coronary intervention in stable and unstable angina. J Cardiol. 2013; 62(2):77-81.

24. Shehata M. Cardioprotective effects of oral nicorandil use in diabetic patients undergoing elective percutaneous coronary intervention. J Interven Cardiol. 2014;27:472-481.

25. Miyoshi T, Ejiri K, Kohno K, et al. Effect of remote ischemia or nicorandil on myocardial injury following percutaneous coronary intervention in patients with stable coronary artery disease: A randomized controlled trial. Int J Cardiol. 2017;236:36-42.

26. Isono T, Kamihata H, Sutani $Y$, et al. Nicorandil suppressed myocardial injury after percutaneous coronary intervention. Int J Cardiol. 2008; 123(2):123-128.

27. Murakami M, Iwasaki K, Kusachi S, et al. Nicorandil reduces the incidence of minor cardiac marker elevation after coronary stenting. Int J Cardiol. 2006;107(1):48-53.

28. Pei WN, Xie RQ, Cui W, et al. Cardiology DO: comparative study for trimetazidine and nicorandil in patients with PCI related myocardial injure. Chinese Circulation J. 2014;29:256-260. Chinese.
Drug Design, Development and Therapy

\section{Publish your work in this journal}

Drug Design, Development and Therapy is an international, peerreviewed open-access journal that spans the spectrum of drug design and development through to clinical applications. Clinical outcomes, patient safety, and programs for the development and effective, safe, and sustained use of medicines are the features of the journal, which

\section{Dovepress}

has also been accepted for indexing on PubMed Central. The manuscript management system is completely online and includes a very quick and fair peer-review system, which is all easy to use. Visit http://www.dovepress.com/testimonials.php to read real quotes from published authors.

Submit your manuscript here: http://www.dovepress.com/drug-design-development-and-therapy-journal 